\title{
Restless legs syndrome: An overview of pathophysiology, comorbidities and therapeutic approaches (Review)
}

\author{
ANDREI VLASIE ${ }^{1}$, SIMONA CORINA TRIFU $^{2 *}$, CRISTIANA LUPULEAC ${ }^{1 *}$, \\ BIANCA KOHN $^{1}$ and MIHAI BOGDAN CRISTEA ${ }^{3 *}$ \\ ${ }^{1}$ Department of Psychiatry, 'Prof. Dr. Alexandru Obregia' Clinical Hospital of Psychiatry, \\ Bucharest 041914; Departments of ${ }^{2}$ Clinical Neurosciences and ${ }^{3}$ Morphological Sciences, \\ 'Carol Davila' University of Medicine and Pharmacy, Bucharest 020021, Romania
}

Received July 5, 2021; Accepted August 5, 2021

DOI: $10.3892 /$ etm.2021.11108

\begin{abstract}
The aim of this overview was to outline the pathophysiology, common comorbidities and current therapeutic modalities in the treatment/management of restless legs syndrome (RLS) a sensorimotor neurological disorder. The main symptom in RLS is a compelling compulsion to move the legs and a sense of restlessness at rest most commonly occurring during the night and improving with movement. The prevalence of secondary RLS among comorbid conditions such as idiopathic pulmonary fibrosis, end-stage renal disease, irritable bowel syndrome and attention deficit/hyperactivity disorder have further elucidated our understanding of the role of the iron-dopamine hypothesis as an etiopathogenetic hallmark in RLS and the efficacy of therapeutic approaches in milder to more severe forms. Currently, RLS treatment uses only symptomatic agents, since a disease-modifying therapy does not yet exist. The phenomena of rebound and augmentation have become central phenomena in overcoming the pharmacotherapeutic challenges when treating with dopaminergic agents in RLS. Considering alternative nonpharmacological therapies, especially for the treatment of RLS in pregnancy has a significant role and positive clinical outcome for patients in controlling symptoms.
\end{abstract}

\section{Contents}

1. Introduction

2. Pathophysiology

Correspondence to: Dr Simona Corina Trifu, Department of Clinical Neurosciences, 'Carol Davila' University of Medicine and Pharmacy, 37 Dionie Lupu Street, Bucharest 020021, Romania E-mail: simona.trifu@umfcd.ro

*Contributed equally

Key words: restless legs syndrome, iron deficiency, dopaminergic neurotransmission, sleep disorder, International Restless Legs Syndrome Study Group, restless leg syndrome risk alleles, pregnancy
3. Diagnostic criteria

4. Restless legs syndrome (RLS)/Willis-Ekbom disease (WED) comorbidities

5. Therapeutic approaches in RLS: Non-pharmacological and pharmacological treatments

6. Conclusions

\section{Introduction}

The authors independently screened literature search results and abstracted data from Clarivate Web of Science (https://www.webofscience.com/wos/woscc/basic-search),Pubmed (https://pubmed.ncbi.nlm.nih.gov/), Scopus (https://www. scopus.com/home.uri) and Google Academic (https://scholar. google.com/) databases having as main research topics the terms: 'Restless leg syndrome' or 'RLS' or 'Willis-Ekbom disease' and 'drugs in RLS' and 'iron deficiency in RLS' and 'treatment of RLS' and 'dopamine'. The research documents published from 2010-2020 were narrowed down, and included articles, studies and cohort studies. Subsequently, an overview of the literature was also performed because some information was older than our proposed time frame and because a large number of articles were combined (since the databases had more than 4,800 articles, including 836 reviews). Studied articles were limited to 100 after the exclusion of similar articles by different authors. The material was categorized to follow the main content of this review.

Restless legs syndrome (RLS), the fourth leading cause of insomnia has a clinical relevance and is often an underdiagnosed medical condition among sleep disorders. An understanding of the etiopathogenesis of RLS by examining both endogenous and exogenous factors, such as CNS dopaminergic and iron deficiency hypotheses as the etiologic hallmarks of RLS has enhanced our clinical understanding of this disorder, and contributed to the improvement of diagnostic and therapeutic approaches. The association of RLS with comorbid conditions such as idiopathic pulmonary fibrosis (IPF), end-stage renal disease (ESRD), irritable bowel syndrome (IBS), attention deficit/hyperactivity disorder (ADHD) and insomnia was further examined. In the evaluation and assessment of patients presenting with RLS symptoms, the 
validated 5-item criteria rating scale, International Restless Legs Syndrome Study Group (IRLSSG) and [The Pittsburgh Sleep Quality Index (BK1)] (PSQI) used to assess the quality of sleep, have revealed the compounding effect of RLS on overall sleep quality, quality of life, and higher prevalence of RLS among the mentioned comorbidities (1). Comorbid anxiety and depression as observed in ESRD, and the treatment of psychiatric complications using various psychotropic medications are important factors which can induce and worsen RLS symptoms (2). As part of a comprehensive array of therapeutic modalities, both pharmacological and nonpharmacological interventions have varying levels of therapeutic outcome in either primary or secondary forms of RLS, with pharmacotherapeutic treatments such as iron supplementation and dopaminergic therapy being more effective in more severe and resistant forms of RLS $(3,4)$.

One hypothesis concerning RLS establishes that underlying the condition there is a regional brain iron deficiency and/or specific genetic factors that disturb the neurotransmission of dopamine in the subcortical brain areas. The pharmacological treatment of RLS is limited to symptomatic medication, since etiological forms of therapy have not been developed. A decrease in RLS symptoms with dopamine agonists, along with the increase of the symptoms when dopamine antagonists that cross the blood-brain barrier are received, as well as the lack of response to domperidone (a compound that does not go through the blood-brain barrier) support the disturbance of dopamine transmission as pathophysiological for this disease. Another important argument for the dopaminergic dysfunction is the decrease in D2 receptors in the striatum. There is a direct association of iron deficiency with a reduced activity of the D2 receptors, while oral iron supplementation proved to be efficient in relieving symptoms of secondary RLS. At present, the main treatment for RLS is represented by dopamine agonists, which are also well tolerated by the patients. Real benefits have also been reported with the use of pramipexol, ropinirole and rotigotine. For instance, ropinirole was well-tolerated and has exhibited actual benefits in RLS patients with associated sleep problems and low quality of life. Other pharmacological resources include clonazepam, used especially for sleep disturbances, as well as gabapentin for the treatment of algic symptoms in RLS (5).

\section{Pathophysiology}

An easily explorable environmental factor (iron), a prominent response to the increasing activity of a neurotransmitter (dopamine) and a well-defined phenotype are the three major pillars that generated a surprisingly complex biology underlying RLS (6).

The genome-wide association studies (GWAS) facilitated by the RLS/Willis-Ekbom disease (WED) well-defined phenotype have identified RLS risk alleles on 5 genomic regions for BTBD9, PTPRD, MAP2k/SKOR1, MEIS1 and TOX3/BC034767 (7-9). Most of these variants have also been associated with the periodic leg movements motor sign (PLMS) of RLS (10). However, the potential genetic pathways to RLS pathophysiology can be narrowed down to two findings: A risk allele on BTBD9 associated with decreased peripheral iron stores (low serum ferritin) and increased PLMS independent of RLS as well as a BDBT9 variant which ties to increased PLMS, RLS diagnoses and excessively reduced peripheral iron stored with blood donations $(11,12)$.

The iron deficiency thesis has been in place since the pioneering studies of Ekbom and Norlander and is supported by epidemiological findings such as a 9 times higher prevalence of RLS in an iron-deficient anemia population than in the general population or the increased risk of RLS in all conditions that compromise iron status (e.g., ESRD, pregnancy) $(6,13)$. Nevertheless, most RLS patients have normal serum ferritin and no indication of peripheral iron deficiency. Rather, a hallmark of RLS appears to be brain iron deficiency, documented by several separate studies which discovered low cerebrospinal fluid (CSF) ferritin in RLS patients $(14,15)$. The brain areas that show iron deficiency include the substantia nigra, and to a lesser degree the putamen, caudate and the thalamus (16). The iron deficiency in these areas is explained by the failure to get enough iron across the blood-brain barrier combined with the failure to import enough iron into critical neuronal cells (e.g., neuromelanin cells of the substantia nigra) (6).

Two major pathophysiological consequences of low brain iron are hypoxia and demyelination (6). Oxygen-transport is dependent on iron, thus a decreased level of iron in the brain should signal hypoxia. It was revealed that hypoxia-inducible factor-1 $\alpha$ (HIF-1 $\alpha)$ was higher in the substantia nigra of RLS patients. In addition, higher than normal HIF- $2 \alpha$ and endothelial growth factor were revealed in the microvessels of an iron-deprived brain (17). A direct consequence of the activation of hypoxic pathways would be the increase of dopaminergic activity, which is indubitable in RLS patients. The hypoxic pathway as a pathway to RLS symptoms would explain the high incidence of RLS in patients with chronic obstructive pulmonary disease (18).

The synthesis of myelin sheaths is also dependent on iron, thus a deficiency in brain iron would lead to less than required myelin; Allen in his review revealed significant decreases in white matter in the corpus collosum, anterior cingulate and precentral gyrus (6). The $25 \%$ drop in myelin identified in postmortem analyses could explain RLS/WED symptoms, especially considering the time-dependent signaling of the sensorimotor integration (19).

The findings of high 3-orthomethyl dopamine (3-OMD) in the CSF correlated with an increased level of the dopamine metabolite, homovanillic acid (HVA), is suggestive of an increase in tyrosine hydroxylase activity leading to high dopamine production $(20,21)$. Furthermore, the decreased fluoro-1-dopa (f-DOPA) uptake suggests a fast turnover of dopamine, consistent with its increased production (6). Thirdly and most importantly, decreased dopamine-transporter (DAT), mostly the membrane-bound one, and decreased D2 receptors were identified in iron-deficient rodents, similar to RLS ones $(22,23)$.

Treating the already excessive brain dopamine in RLS with levodopa would appear counterintuitive, but the key to understanding it lies in the circadian rhythm of both dopaminergic activity and RLS/WED symptoms. The increase of dopamine activity leads to a natural downregulation at both the receptor and intracellular level. However, dopamine has a clear circadian rhythm increasing in the morning and decreasing in the 
evening and night. In these circumstances, the post-synaptic response in RLS is adequate for daytime but exaggerated for nighttime, resulting in a nighttime dopaminergic deficit despite the overall surplus of dopamine. This dynamic explains the circadian pattern of nighttime RLS symptoms, alternating with arousal and alertness in the morning that prevents the expected sleepiness of a fragmented RLS sleep (6). Administering a low dose of dopamine at night may be an apparent relief for the patient but it goes in a slippery-slope mechanism of increasing the consequent downregulation, thus rendering the RLS symptoms worse. This is an example of tolerance at first, with the patient needing increasing doses for the same effect, but actually it also becomes an augmentation, worsening the underlying pathology. The adjustment of the system to increasing doses of dopamine ultimately renders the symptoms of RLS worse, with the patient struggling with daytime symptoms and becoming dependent on medication to avoid withdrawal $(6,24)$. The key here is to use long-acting medication such as transdermal rotigotine in order to reduce the risk of debilitating augmentation. In addition, low doses of medication are indicated for a reduced augmentation effect even with the long-acting products, rendering dopamine medication a limited line of therapy for RLS (25).

A total of 13 different genes implicated in RLS have been identified as single nucleotide polymorphisms on chromosomes 6p21.2 (BTBD9), 2p14 (MEIS1), 9p24.1-p23 (PTPRD), 15q23 (MAP2K5/SKOR1) and 16q12.1 (TOX3/BC034767) based on GWAS. In addition, several possible loci have been identified on chromosomes 12q12-q21, RLS2 on 14q13-q21, RLS3 on 9p24-p22, RLS4 on 2q33, RLS5 on 20p13, RLS6 on $19 \mathrm{p} 13$, and RLS7 on 16p12.1 (26,27). The functions of these genes are in close connection to the neurological development of the embryonic limbs even if the pathophysiological functions are not yet clear.

\section{Diagnostic criteria}

Even though RLS/WED was firstly approached as a nosological entity in 1945 by Karl-Axel Ekbom (28) and several diagnostic criteria have been used throughout the years, a final consensus was attained only in 2012. In 2008, the IRLSSG and WED Foundation organized and sponsored sessions of clinical workshops and workgroups which were held in Baltimore, MD at the John Hopkins Mount Washington Conference Center. Members of IRLSSG and experts from other fields addressed the need of revision of the 2003 criteria, given the complex and broad etiology and symptomatology of the disease. Their conclusions and suggestions were then assembled by the chairman of the workshop and this was followed by a lengthy process of dialogue, analysis and adjustments by other members of IRLSSG, as well as researchers and clinicians from around the world. The final version became official in 2012, when it was published on the IRLSSG website (www. irlssg.org).

As no biomarkers or paraclinical investigations are currently available for the diagnosis of this disorder, the clinician has the key role in providing an adequate anamnesis and underlining the signs and symptoms which match the current formal criteria; five important diagnostic criteria that have to be met.
The first criterion remained the same as in 2003 and it is defined by 'an urge to move the legs'. Even though this often co-occurs with a feeling of 'unpleasant sensations in the legs', 'the urge to move' is mandatory and sufficient for an RLS diagnosis.

Despite the name of the syndrome, the urge to move may also be present in other body parts: arms (21-57\%) or in more severe forms even the torso and hips; the face is rarely involved (29-34).

For an RLS/WED diagnosis, leg involvement is required. There are reports of cases with predominant involvement of the arms, but their small number got them listed by the IRLSSG as variant of the disorder and thus are not included in an extended definition $(35,36)$.

'An urge to move the legs' means that in a typical presentation, both legs are affected; but this does not always happen symmetrically or simultaneously. A simple unilateral presentation requires a rigorous differential diagnosis with a unilateral neurological condition.

Regarding the location on the legs, the most affected areas appear to be the middle parts of the calves and thighs $(28,33)$. The presence of a progression pattern in time (or during the same episode) could be an indicator of a more severe evolution or spreading to other body parts.

Another essential feature of RLS is the appearance or aggravation of unpleasant sensations correlated with the need to move the legs, in moments of calm, rest or inactivity corresponding to the sitting or lying position. These sensations occur or get worse in the evening then during the night time.

Rest or lack of activity starts or aggravates the symptoms. This feature was highlighted by research based on the suggested immobilization test paradigm; a study conducted by Michaud et al (37).

Another criterion is based on the disappearance or reduction of unpleasant sensations or the desire to move the legs while the patient is performing a motor activity, a movement; this effect is maintained throughout this activity. The only difference in this criterion compared with the one from 2003 is the pairing between the 'urge to move' and 'unpleasant sensations'.

The professional conducting the assessment must pay attention to these 3 features: relief vs. resolution, immediacy and persistence. These concepts may be troubling for patients, as for example, a patient who obtains relief only during running, may report that movement does not help them, only because the symptoms return as soon as the individual stops this activity. However, it must also be taken into consideration that in very severe cases, the physical activity may bring little to no relief.

A specific feature of RLS is that the symptoms tend to exacerbate in the evening and during the night. An improvement can be felt in the state of deep sleep or early in the morning, while mornings are usually symptom-free. This predictable variation during $24 \mathrm{~h}$ appears without being related to other mentioned factors such as activity, rest or sleep deprivation (38-41).

One key question in the assessment process should aim to identify the difference between the intensity of the symptoms during resting time when comparing mornings with evenings. RLS/WED patients will have worse symptoms during the 
inactivity periods in the evenings. Patients with more severe presentations of RLS/WED may not exhibit important variations of symptoms during the day, but they must be asked if these existed when their symptoms used to be milder (41).

Other medical or behavioral conditions including myalgia, leg edema, leg cramps, venous stasis, arthritis and positional discomfort can lead to similar symptoms as RLS, but these conditions should not only be correlated with RLS.

This new criterion is added with the intention of expanding the specificity of the diagnosis. As numerous diseases mimic RLS/WED by meeting the first 4 criteria, adding the problem of differential diagnosis on the formal criteria list will improve the success rate of diagnosis especially in written scales and surveys, leading the success rate of these assessments closer to the $90 \%$ accuracy obtained by clinicians (42-44).

This does not exclude the fact that patients with RLS/WED may have other comorbidities with shared features (e.g., peripheral neuropathy and alcohol) $(45,46)$.

\section{Restless legs syndrome (RLS)/Willis-Ekbom disease (WED) comorbidities}

$I P F$. IPF is a progressive, chronic, fibrotic lung disease with a bleak overall prognosis of 2-3 years and multiple comorbidities, including cardiovascular disease, chronic obstructive pulmonary disease (COPD), gastroesophageal reflux disease (GERD), lung cancer but also sleep disorders such as nocturnal hypoventilation or obstructive sleep apnea syndrome (OSAS) $(47,48)$. In a debuting, recent study by Varone et al a cohort of 50 IPF patients was evaluated for the presence of RLS using the validated 5-item criteria, its severity using the IRLSSG rating scale and the PSQI to assess the quality of their sleep (1). The results revealed a 2.5 higher prevalence of RLS in IPF patients compared with the controls, compounding the RLS effect on the already poor sleep quality of the patients (1). The study thus identified RLS as a new, frequent comorbidity to IPF with a devastating effect on the sleep quality of the patients, making the dopaminergic medication or other forms of therapy a possible solution to improved sleep and life-quality of the IPF patients (1).

$E S R D$. RLS has an important prevalence (up to 68\%) in the ESRD population with dire implications ranging from low sleep and quality of life to comorbid anxiety, depression and, if left untreated, to potentially lethal cardiovascular diseases (49-51).

Ohayon et al revealed ethnic and age-related differences within the ESRD population with the RLS comorbidity comprised of a higher prevalence of RLS in European and American populations compared with Asians, as well as an age-related increase in prevalence for Europeans and Americans that is not is not found in the Asian population (52). Nevertheless, the whole spectrum of chronic kidney disease (CKD) patients, especially those undergoing hemodialysis (HD) and continuous ambulatory peritoneal dialysis (CAPD) have higher prevalence of RLS compared with the general population. Several studies have tried to identify parameters (such as age, weight, BMI or even serum hemoglobin or ferritin levels) that would predict an increased risk for RLS in ESRD but failed to render consistent results (53-55). Not even the severity of renal failure could be singled out as a predicting factor for RLS because not all ESRD patients that received renal transplants were relieved of RLS subsequently (56). A single study has identified as predictors of the risk for RLS/WED both the positive family history for RLS and reduced/absent renal function and peripheral neuropathy (57).

With RLS related to ESRD being considered a secondary form of RLS, there are no differences in clinical manifestation between primary and secondary forms of RLS. There are some clinical aspects to be considered when diagnosing RLS in ESRD: Uremic and diabetic neuropathy are frequent in ESRD patients manifesting as prickling sensations in the lower extremities with little circadian variability, thus clinicians/doctors should be aware not to misdiagnose them by resorting to neurological examinations (2). Moreover, the evaluation of respiratory events (such as apnea) and motor events (such as limb movements) is assisted through a polysomnography recording considering that sleep apnea is highly comorbid with ESRD (2).

Before therapeutic interventions, one should note that numerous substances, mainly psychotropic medication (neuroleptics, tricyclics, SSRIs, SNRIs) can induce or worsen RLS and are frequently prescribed for the psychiatric complications of ESRD (2).

Regarding the pharmacological treatment in ESRD patients with RLS, the list of drugs used is similar to that for primary RLS/WED consisting of: levodopa, dopamine agonists, GABA agonists and intravenous iron (2). In two different studies gabapentin proved superior to levodopa, despite the not very stringent study design $(58,59)$. As far as the time of administration, $2 \mathrm{~h}$ before bedtime proved optimal for all drugs except for intravenous iron and gabapentin, that was administered in an improved way after HD sessions, 3 times per week (2). Apart from these conventional medications, previous studies investigating antioxidant vitamin supplementation with vitamin $\mathrm{C}, \mathrm{E}$ or both revealed positive results, with an increase in the IRLS score up to $50 \%(60,61)$.

A non-pharmacological researched approach identified that intradialytic aerobic exercise training during HD 3 times per week can be as effective as a low-dosage dopamine agonist for RLS $(2,62)$.

IBS. IBS represents a bowel disorder mainly affecting the function of the bowel, with no current tools at the disposal of physicians to detect any structural or biochemical abnormalities of this condition. Patients usually describe the following symptoms: Discomfort or even pain in the abdominal area, a different color pattern and regularity of stool and frequent bloating. The severity of the symptoms may vary, from completely debilitating, to moderate or mild symptoms. The irritable bowel syndrome is usually associated with other comorbidities (non-psychiatric/psychiatric) such as: RLS, pain syndromes, headaches, migraines and overactivity of the bladder. The psychiatric evaluation includes: depression and anxiety. The prevalence of IBS is high $(\sim 12 \%)$ and the disorder highly affects the quality of life (63). Revealing the link between IBS and RLS can improve our understanding of the pathophysiology of IBS, especially since it represents a somewhat mystery of the neuro-gastroenterology field and it is also the most common functional bowel disorder. 
$A D H D$. ADHD occurs before the age of 12 and is an inherited neurological developmental disorder that has a common pathology with RLS in the central nervous system. Previous studies correlating sleep disorders with ADHD pathology have led to the identification of functional cortical areas involved in both pathologies: Attention (64), inhibition (65) and working memory. Moreover, there is a positive correlation between ADHD and sleep disorders, including the manifestation of similar symptoms (66).

Children who are diagnosed with both RLS and ADHD appear to face a certain spectrum of symptoms that overlap such as: Alterations of the diurnal activities, inattention (if the child is diagnosed with attention deficit disorder (ADD), the hyperactivity symptom is not present), difficulties in mood regulation, paroxysms of hyperactivity and all leading, in most cases, to poor school performance.

The same studies correlate sleep disorders caused by RLS with attention deficits as a secondary factor for these conditions or assume the existence of molecular mechanisms involved in both disorders. Furthermore, the dopaminergic deficit should be taken into consideration when it comes to the pathogenesis of both RLS and ADHD.

Insomnia. The International Classification of Sleep Disorders-Third Edition (ICSD-3) defines insomnia as: 'Difficulty initiating sleep, maintaining sleep, or waking up too early; presence of these symptoms despite adequate opportunity for sleep; and daytime deficits'. The difference between acute and chronic insomnia lays in the period of time of which patients experience the aforementioned symptoms. In the case of acute insomnia, the symptoms last less than three months and more than three months for chronic insomnia (occurring more than three times a week). Acute insomnia is usually linked to a stressor or event that led to insomnia in the first place (67).

The symptoms that appear in the RLS, such as uncontrollable urges to move the limbs that get worse at rest usually appear during the night time or the evening. The fact that the symptoms appear during the second part of the day, mainly at nighttime affects the ability to initiate or continue the sleep cycle. RLS is considered to be the fourth leading cause of insomnia.

\section{Therapeutic approaches in RLS: Non-pharmacological and pharmacological treatments}

\section{Pharmacotherapy}

General principles. The individualization of the treatment in RLS must respect the following principles: It starts from the minimum effective dose, and this will be increased with caution and only if necessary, with knowledge on the personality structure of these patients, clinical interference with neuromuscular pathology, receptor sensitivity, as well as the sensitive elements of personality, which render the individual in question more receptive to side effects. The second principle is that medication should be initiated early enough after diagnosis to prevent habituation to suffering and self-induction of a pattern that, at the limit, resembles hypochondria. The third principle is related to the cyclicity of sleep, the doses being necessary to be administered in the evening, in a single dose or in two divided doses, the first one frequently before the evening meal and the second late in the night to ensure the quality of sleep, shortening REM periods and controlling the nocturnal aggravation phenomenon. The fourth principle is established when it is necessary to give up monotherapy and supplementation with a second pharmaceutical agent with different receptor action (3).

Unfortunately, the current pharmacological approaches are focused on symptomatology, when new lines should be opened in the direction of modulatory therapies and those that address causation. The risks that may occur in the long term are generated by the natural progression of the disease itself, the development of drug tolerance and by the psychological management, with characteristics including depression, increased sensitivity and emotional lability, feeling helpless, feelings of uselessness and incurability, which can evolve to comorbidity with a depressive episode of moderate or severe intensity. Subjects complain throughout life of the accumulation of extra time spent in suffering and occasionally of therapeutic alliances that decrease in intensity because specialists become discouraged with the patient if they fail to control RLS and its main comorbidities including hypochondria and depression (68).

Clinical situations that impose the need for pharmacological treatment. Medication needs to be instituted when the symptoms are clinically significant, when they occur with frequency and severity affecting quality of life. What has been previously described belongs to the chronic form of RLS as opposed to the occasional form, when treatment could be taken intermittently $(9,11)$. Of choice are the dopaminergic agonists aforementioned, pramipexol and ropinirole, which are also used in Romania. The specialized literature also mentions use of the rotigotine patch with which there is no experience in Romania. Dopaminergic agonists act quickly by minimizing the symptoms even at low doses, but, paradoxically, if the aspect of control escapes the care of the patient or specialist, in the sense of an unjustified increase, the worsening of the severity of the disease will be faced. Pramipexol and ropinirole are commonly used to treat Parkinson's, but the doses addressed in RLS are markedly lower (5).

The administration of iron should not be neglected and its dosage should be gradually increased (69). Serum iron is routinely measured (a more complex battery that includes ferritin, sideremy, transferrin and iron-binding capacity), but what really matters in RLS is not the level of blood iron but the amount of iron in the synaptic cleft being directly related to RLS symptoms. However, usual dosages claim that if serum ferritin $\leq 75 \mu \mathrm{g} / 1$ or the transferrin and iron-binding capacity is $<20 \%$ it becomes imperative to administer oral iron therapy. In case of gastric intolerance, nausea or fever, intravenous iron preparations such as ferric carboxymaltose in high doses or low molecular weight dextran iron can be used $(4,70,71)$. Specialists should be careful when considering iron therapy, checking the ratio of serum ferritin measured in $\mu \mathrm{g} / \mathrm{l}$ to the age of the patient. In other words, iron should be given if the ferritin value is less than the age of the patient (21). The iron used intravenously must be chosen at the right time, namely when the ferritin is not extremely low, so that the total iron-binding capacity can fulfill its function and the absorption of iron in the intestinal 
transit is efficient. Under normal physiological conditions, even if the patient receives unjustified iron medication, there is an endogenous regulation in terms of oral absorption of iron, which prevents its accumulation in excess (69,71-73).

Dopaminergic therapy. In 1982 Akpinar et al revolutionized RLS management by discovering the effectiveness of levodopa (74). This is a carboxylase doping inhibitor that immediately and markedly relieves symptoms (3,75). Following the study by Akpinar $e t$ al, which investigated patients diagnosed with intermittent RLS, another study (75) was performed with multiple dopaminergic agents, which were considered effective in RLS and peripheric limb movement disorder. Levodopa is well tolerated in subjects with RLS, and dyskinesias induced in Parkinson's patients are not found in subjects with RLS. If dopaminergic treatment lasted $>5$ years, nigrostriatal binding capacity was normalized according to PET-type studies performed with 18F-Fluorodopa (76). The aforementioned research confirmed the additional safety of this long-term medication. Unfortunately, the negative aspects remain: The risk of relapse (regardless of how long the pharmacological treatment lasted), as well as the risk of developing tolerance, in other words the need to increase the doses. Relapse can be considered a consequence of the fact that levodopa is a medicine with a short half-life. Patients themselves end up shortening their time between doses gradually to $5 \mathrm{~h}$, then to $4 \mathrm{~h}$, which potentiates tolerance and early recurrence of symptoms. The problem could be solved by supplementing the medication with a prolonged-release medicine. It would be desirable to administer it in the morning, although the effectiveness of the protocol is not yet fully supported by enough evidence-based medicine studies (3,77).

Modern changes have occurred in the pharmacological approach related to the use of levodopa, which in the past was considered the treatment of choice, and currently proves effective only in patients with intermittent symptoms (even if they are severe) (3). Orientation to other medicines to the detriment of levodopa is based on the following clinical considerations: Up to $80 \%$ of patients treated exclusively with levodopa for RLS exhibit increased symptoms within several months, of which one third of them reach severe symptoms that curl depression as comorbidity or hypochondriac concerns or suicidal ideation. This imposes a firm need to change the therapy (78). The need to increase doses is a serious problem that involves the following aspects: Symptoms appear earlier in the day, become more severe, rest time is significantly reduced, new areas of the body appear in which symptoms are felt and which were not previously involved.

In principle, dopaminergic drugs are at risk of increasing doses, but of these, the phenomenon is most common in levodopa (up to 73\%) and less common in dopamine agonists. In the last-mentioned case, the need to increase doses occurs in $10 \%$ of cases requiring short-term medication and in $42-68 \%$ in patients with over 10 years of treatment $(69,70)$. Short-acting dopamine agonists, such as ropinirole and pramipexole, have a higher risk of short-term dose escalation compared with those with a longer half-life (transdermal rotigotine). In the case of the second category (dopaminergic agonists with a longer half-life) a lower circadian fluctuation of serum dopamine levels is observed. In other words, dopaminergic receptors are stimulated, but in a less pulsating manner, allowing downward regulation of dopamine receptors, which in turn reduces circadian variation $(69,70,79-81)$. A pertinent question remains whether the continuous administration of a dopaminergic antagonist for $24 \mathrm{~h}$ treats the symptom or simply masks the symptoms or does nothing but risk the development of tolerance and require the body to increase the doses.

Methods to increase RLS treatment in case of therapeutic resistance. Due to the existence of multiple comorbidities, numerous of them psychiatric, the increase can be performed gradually or in a sudden manner in a hospital, if the severity of symptoms requires it (82) and with an efficient management of the therapeutic alliance. Meetings of 30-min duration are preferred, in which the subjects are listened to with their somatic complaints, in an active and empathetic manner, without being contradicted; the depressive component can be recognized and brought into discussion, without denying their moral and physical suffering and without appealing to the notion of hypochondria. In critical moments, such meetings must be reserved for a few days. If the suffering of the patient is mild to moderate, and the patient is already being treated with dopaminergic agents, the following strategies are possible: Increase the dose or administer it earlier or split it into two doses, both administered in the evening. If the suffering is clinically significant, transition from a short-acting dopamine agonist to an $\alpha 2 \delta$ ligand is suggested. In the case of severe RLS, an attempt is made to relieve the patient of previous medication, as fast as possible, which should not exceed 10 days. Subsequently, the transition will be made from a long-acting dopamine agonist to one with an even longer-lasting action or to an $\alpha 2 \delta$ ligand (83). If ferritin is $<100 \mu \mathrm{g} / \mathrm{l}$ then these are characterized as severe cases that require intravenous iron infusion and if the clinical symptoms bring with them a pain that is difficult to tolerate, opioids are selected, which act on GABAergic receptors and have a quick response; among the beneficial effects, along with the removal of pain is a relative state of euphoria.

$R L S$ approach in pregnancy. For a first stage, non-pharmacological treatments are desired; it is considered that performing moderate to intense physical activities, with attention to the risk of abdominal trauma and at the chosen time of day, when physical exercises should be performed (if sport is practiced in the evening, it can cause insomnia, which in turn will aggravate the symptoms of RLS) have an important role $(80,84)$. From the pharmacological point of view, oral supplementation with iron is selected, or the intravenous method if the ferritin level falls $<75 \mu \mathrm{g} / \mathrm{l}$. If the aforementioned are not sufficient to alleviate the symptoms, the carbidopa/levodopa dopaminergic combination will be used as a necessary option in a daily dose of 20/100 to $50 / 200 \mathrm{mg}$. Opting for a prolonged-release formula reduces the risk of developing tolerance on the medication. Dopaminergic agonists are contraindicated in pregnancy. Due to the inhibition of prolactin, breastfeeding is not triggered because the dopaminergic mechanisms are inhibited by medication. However, if the treatment is interrupted, this aspect is reversible $(80,84)$. 
Other medications used in RLS

Antiepileptic drugs. A randomized study on the effectiveness of ameliorating paresthesia and reducing the number of RLS attacks, demonstrates the effectiveness of carbamazepine at a dose of 100-400 mg, which is given at bedtime, to demonstrate its effectiveness at night $(44,85)$. Although the effectiveness of carbamazepine is certain, given the side effects which are mainly of hepatic involvement (with up to a 3 times increase in transaminases), but also the risk of spinal cord depression, recently, gabapentin is preferred, which also appears to be effective and tolerated in an improved way (86). Pregabalin, lamotrigine or topiramate may also have a special role in patients who report painful paresthesia or underlying neuropathies. The use of antiepileptics in RLS is performed at lower doses compared with those in bipolar affective disorders and significantly lower than those in seizures (87).

Opioids. Opioids were first described as being used in RLS in a double blinded study, by Walters et al. This research group conducted a controlled study on oxycodone that improved the quality of life of patients by its symptomatic action, reducing periodic limb movement disorder (88). It is well known that opioids also have a high risk of addiction and should be avoided in patients whose personality structure has such inclinations or who risk using them to instantly eliminate depression and feelings of euphoria. Thus, this class of drugs remains preserved for refractory cases (89).

Benzodiazepines. Unfortunately, double-blind cross-over studies have been performed on a small number of patients (90). Clonazepam has been considered the first choice due to the mechanism that acts on the one hand, as a muscle relaxant and on the other, as a hypno-inducer (91). The risks of sedation and nocturnal falls should not be overlooked if therapeutic doses are not well controlled, if the patient is elderly, or at risk of addiction or uses poly-medication.

Non-pharmacologic interventions. In a first stage they can be applied singularly, and in a later stage they will remain in the background, in various pharmacological combinations, to relieve the symptoms. Roundly, the general instructions are based on the following indications: The combination of alcohol, caffeine, nicotine or activator-type antidepressants should be minimized. Exercise should remain at a moderate level. The body must be given proper care by applying warmth or hot baths, massage at the lower and/or upper limbs, maintaining healthy habits in terms of inducing sleep by maintaining a regular schedule, learning relaxation and meditation exercises to be used at night and avoidance of forced sleep at any cost. Subjects are advised to engage in motivational coaching and/or cognitive-behavioral therapy and to develop their own warning strategies when their own behavior becomes sedentary. Pneumatic foot compression, near-IR light spectroscopy (NIRS) have also been used, although there is insufficient evidence of their efficacy as adjuvants to pharmacological treatments $(67,69,92)$. Complementary therapies such as acupuncture, transcranial magnetic stimulation, vibration equipment applied to muscles during exercise, cryotherapy and aromatherapy should not be overlooked $(67,93,94)$. Antipsychotics have the potential to increase the symptoms of RLS or periodic limb movement disorder and upon pertinent evaluation, the clinician is required to make an effective differential diagnosis with hypochondria (or even delusional somatic disorder) to avoid this group of drugs (3). Even antihistamines can worsen RLS symptoms. Unfortunately, exclusive non-pharmacological approaches are presented in randomized studies without well-defined control groups and suffer from a lack of clarity in the objective analysis of the result. To all these aforementioned symptoms, poor sleep quality is also added $(67,94)$. Non-pharmacological options remain effective for mild cases $(68,69)$.

\section{Conclusions}

A multifactorial understanding of the etiopathogenesis of RLS with regards to primary and secondary forms and its prevalence in comorbid conditions such as IPF, ESRD, IBS and ADHD have brought greater attention to the clinical relevance of this sensorimotor neurological disorder and in the development of rating scales such as IRLSSG. In addressing the individual therapeutic needs and the clinical outcome of the patient, by understanding the variant or form of RLS postdiagnosis, and the level of chronicity and distress of the illness overtime for the patient, clinicians can successfully work together with their patients in managing the symptoms of RLS by implementing various therapeutic modalities i.e., pharmacologic vs. non-pharmacologic. In this overview, the iron-dopamine hypothesis postulates that RLS involves a regional iron deficiency affecting the neurotransmission of dopamine in the brain subcortical area, and/or by genetic factors affecting the iron metabolism in the brain. Understanding the role of iron and the dopaminergic system are, therefore, essential aspects in the therapeutic management in idiopathic and secondary RLS. The prevalence of panic disorder, generalized anxiety disorder, major depression and other psychiatric disorders in patients with a diagnosis of RLS is of clinical relevance, encouraging stronger clinical assessment of RLS symptomatology perhaps in the treatment and diagnosis of psychiatric disorders.

\section{Acknowledgements}

Not applicable.

\section{Funding}

No funding was received.

\section{Availability of data and materials}

Not applicable.

\section{Authors' contributions}

SCT and AV designed and drafted the initial review. CL and BK gathered the medical information and produced the final presentation and structure of the review. CL, BK and AV investigated the present area of research and gathered relevant and important information. SCT and MBC finalized the work and approved the final version of the review. AV and CL 
confirm the authenticity of all the raw data. All authors read and approved the final manuscript.

\section{Ethics approval and consent to participate}

Not applicable.

\section{Patient consent for publication}

Not applicable.

\section{Competing interests}

The authors declare that they have no competing interests.

\section{References}

1. Varone F, Friello L, Di Blasi C, Sgalla G, Luigetti M, Iovene B, Richeldi L, Della Marca G and Brunetti V: Restless legs syndrome: A new comorbidity in idiopathic pulmonary fibrosis. Respir Med 170: 105982, 2020.

2. Tachibana N: Living with restless legs syndrome/willis-ekbom disease. Sleep Med Clin 10: 359-367, xv, 2015.

3. Cotter PE and O'Keeffe ST: Restless leg syndrome: Is it a real problem?. Ther Clin Risk Manag 2: 465-475, 2006.

4. Allen RP, Picchietti DL, Auerbach M, Cho YW, Connor JR, Earley CJ, Garcia-Borreguero D, Kotagal S, Manconi M, Ondo W, et al: Evidence-based and consensus clinical practice guidelines for the iron treatment of restless legs syndrome/Willis-Ekbom disease in adults and children: An IRLSSG task force report. Sleep Med 41: 27-44, 2018.

5. Buchfuhrer MJ: Strategies for the treatment of restless legs syndrome. Neurotherapeutics 9: 776-790, 2012.

6. Allen RP: Restless leg syndrome/willis-ekbom disease pathophysiology. Sleep Med Clin 10: 207-214, xi, 2015.

7. Schormair B, Kemlink D, Roeske D, Eckstein G, Xiong L, Lichtner P, Ripke S, Trenkwalder C, Zimprich A, Stiasny-Kolster K, et al: PTPRD (protein tyrosine phosphatase receptor type delta) is associated with restless legs syndrome. Nat Genet 40: 946-948, 2008.

8. Winkelmann J, Czamara D, Schormair B, Knauf F, Schulte EC, Trenkwalder C, Dauvilliers Y, Polo O, Högl B, Berger K, et al: Genome-wide association study identifies novel restless legs syndrome susceptibility loci on 2p14 and 16q12.1. PLoS Genet 7: e1002171, 2011.

9. Winkelmann J, Schormair B, Lichtner P, Ripke S, Xiong L, Jalilzadeh S, Fulda S, Pütz B, Eckstein G, Hauk S, et al: Genome-wide association study of restless legs syndrome identifies common variants in three genomic regions. Nat Genet 39: 1000-1006, 2007.

10. Moore H IV, Winkelmann J, Lin L, Finn L, Peppard P and Mignot E: Periodic leg movements during sleep are associated with polymorphisms in BTBD9, TOX3/BC034767, MEIS1, MAP2K5/SKOR1, and PTPRD. Sleep 37: 1535-1542, 2014.

11. Stefansson H, Rye DB, Hicks A, Petursson H, Ingason A, Thorgeirsson TE, Palsson S, Sigmundsson T, Sigurdsson AP, Eiriksdottir I, et al: A genetic risk factor for periodic limb movements in sleep. N Engl J Med 357: 639-647, 2007.

12. Vilariño-Güell C, Soto AI, Young JE, Lin SC, Uitti RJ, Wszolek ZK and Farrer MJ: Susceptibility genes for restless legs syndrome are not associated with Parkinson disease. Neurology 71: 222-223, 2008.

13. Allen RP, Auerbach S, Bahrain H, Auerbach M and Earley CJ: The prevalence and impact of restless legs syndrome on patients with iron deficiency anemia. Am J Hematol 88: 261-264, 2013.

14. Earley CJ, Connor JR, Beard JL, Malecki EA, Epstein DK and Allen RP: Abnormalities in CSF concentrations of ferritin and transferrin in restless legs syndrome. Neurology 54: 1698-1700, 2000.

15. Mizuno S, Mihara T, Miyaoka T, Inagaki T and Horiguchi J: CSF iron, ferritin and transferrin levels in restless legs syndrome. J Sleep Res 14: 43-47, 2005.
16. Rizzo G, Manners D, Testa C, Tonon C, Vetrugno R, Marconi S, Plazzi G, Pizza F, Provini F, Malucelli E, et al: Low brain iron content in idiopathic restless legs syndrome patients detected by phase imaging. Mov Disord 28: 1886-1890, 2013.

17. Patton SM, Ponnuru P, Snyder AM, Podskalny GD and Connor JR: Hypoxia-inducible factor pathway activation in restless legs syndrome patients. Eur J Neurol 18: 1329-1335, 2011.

18. Benediktsdottir B, Janson C, Lindberg E, Arnardóttir ES, Olafsson I, Cook E, Thorarinsdottir EH and Gislason T: Prevalence of restless legs syndrome among adults in Iceland and Sweden: Lung function, comorbidity, ferritin, biomarkers and quality of life. Sleep Med 11: 1043-1048, 2010.

19. Connor JR, Ponnuru P, Lee BY, Podskalny GD, Alam S, Allen RP, Earley CJ and Yang QX: Postmortem and imaging based analyses reveal CNS decreased myelination in restless legs syndrome. Sleep Med 12: 614-619, 2011.

20. Allen RP, Connor JR, Hyland K and Earley CJ: Abnormally increased CSF 3-Ortho-methyldopa (3-OMD) in untreated restless legs syndrome (RLS) patients indicates more severe disease and possibly abnormally increased dopamine synthesis. Sleep Med 10: 123-128, 2009.

21. Stiasny-Kolster K, Möller JC, Zschocke J, Bandmann O, Cassel W, Oertel WH and Hoffmann GF: Normal dopaminergic and serotonergic metabolites in cerebrospinal fluid and blood of restless legs syndrome patients. Mov Disord 19: 192-196, 2004.

22. Erikson KM, Jones BC, Hess EJ, Zhang Q and Beard JL: Iron deficiency decreases dopamine $\mathrm{D} 1$ and $\mathrm{D} 2$ receptors in rat brain. Pharmacol Biochem Behav 69: 409-418, 2001.

23. Erikson KM, Jones BC and Beard JL: Iron deficiency alters dopamine transporter functioning in rat striatum. J Nutr 130: 2831-2837, 2000 .

24. Trifu SC, Tudor A and Radulescu I: Aggressive behavior in psychiatric patients in relation to hormonal imbalance (Review). Exp Ther Med 20: 3483-3487, 2020.

25. Oertel W, Trenkwalder C, Beneš H, Ferini-Strambi L, Högl B, Poewe W, Stiasny-Kolster K, Fichtner A, Schollmayer E, Kohnen R, et al: Long-term safety and efficacy of rotigotine transdermal patch for moderate-to-severe idiopathic restless legs syndrome: A 5-year open-label extension study. Lancet Neurol 10: 710-720, 2011.

26. Desautels A, Turecki G, Montplaisir J, Xiong L, Walters AS, Ehrenberg BL, Brisebois K, Desautels AK, Gingras Y, Johnson WG, et al: Restless legs syndrome: confirmation of linkage to chromosome 12q, genetic heterogeneity, and evidence of complexity. Arch Neurol 62: 591-596, 2005.

27. Yang Q, Li L, Chen Q, Foldvary-Schaefer N, Ondo WG and Wang QK: Association studies of variants in MEIS1, BTBD9, and MAP2K5/SKOR1 with restless legs syndrome in a US population. Sleep Med 12: 800-804, 2011.

28. Ekbom $\mathrm{K}$ and Ulfberg J: Restless legs syndrome. J Intern Med 266: 419-431, 2009.

29. Bassetti CL, Mauerhofer D, Gugger M, Mathis J and Hess CW: Restless legs syndrome: A clinical study of 55 patients. Eur Neurol 45: 67-74, 2001.

30. Winkelmann J, Wetter TC, Collado-Seidel V, Gasser T, Dichgans M, Yassouridis A and Trenkwalder C: Clinical characteristics and frequency of the hereditary restless legs syndrome in a population of 300 patients. Sleep 23: 597-602, 2000.

31. Holmes R, Tluk S, Metta V, Patel P, Rao R, Williams A and Chaudhuri KR: Nature and variants of idiopathic restless legs syndrome: observations from 152 patients referred to secondary care in the UK. J Neural Transm (Vienna) 114: 929-934, 2007.

32. Michaud M, Chabli A, Lavigne G and Montplaisir J: Arm restlessness in patients with restless legs syndrome. Mov Disord 15: 289-293, 2000.

33. Karroum EG, Leu-Semenescu S and Arnulf I: Topography of the sensations in primary restless legs syndrome. J Neurol Sci 320: 26-31, 2012.

34. Buchfuhrer MJ: Restless legs syndrome (RLS) with expansion of symptoms to the face. Sleep Med 9:188-190, 2008.

35. Ruppert E, Cretin B, Meyer C, Kilic-Huck U and Bourgin P: Characterization of periodic upper limb movement disorder in a patient with restless arms syndrome. Mov Disord 27: 1459-1461, 2012.

36. Horvath J, Landis T and Burkhard PR: Restless arms. Lancet 371: $530,2008$.

37. Michaud M, Lavigne G, Desautels A, Poirier G and Montplaisir J: Effects of immobility on sensory and motor symptoms of restless legs syndrome. Mov Disord 17: 112-115, 2002. 
38. Duffy JF, Lowe AS, Silva EJ and Winkelman JW: Periodic limb movements in sleep exhibit a circadian rhythm that is maximal in the late evening/early night. Sleep Med 12: 83-88, 2011.

39. Hening WA, Walters AS, Wagner M, Rosen R, Chen V, Kim S, Shah $\mathrm{M}$ and Thai O: Circadian rhythm of motor restlessness and sensory symptoms in the idiopathic restless legs syndrome. Sleep 22: 901-912, 1999.

40. Trenkwalder C, Winkelmann J, Inoue Y and Paulus W: Restless legs syndrome-current therapies and management of augmentation. Nat Rev Neurol 11: 434-445, 2015.

41. Trenkwalder C, Hening WA, Walters AS, Campbell SS, Rahman K and Chokroverty S: Circadian rhythm of periodic limb movements and sensory symptoms of restless legs syndrome. Mov Disord 14: 102-110, 1999.

42. Möller C, Wetter TC, Köster J and Stiasny-Kolster K: Differential diagnosis of unpleasant sensations in the legs: Prevalence of restless legs syndrome in a primary care population. Sleep Med 11: 161-166, 2010.

43. Hening WA, Allen RP, Washburn M, Lesage S and Earley CJ: Validation of the Hopkins telephone diagnostic interview for restless legs syndrome. Sleep Med 9: 283-289, 2008.

44. Benes $\mathrm{H}$ and Kohnen R: Validation of an algorithm for the diagnosis of restless legs syndrome: The restless legs syndrome-diagnostic index (RLS-DI). Sleep Med 10: 515-523, 2009.

45. Hening WA, Allen RP, Washburn M, Lesage SR and Earley CJ: The four diagnostic criteria for restless legs syndrome are unable to exclude confounding conditions ('mimics'). Sleep Med 10: 976-981, 2009.

46. Trifu S, Carp EG and Nadoleanu A: Alcohol as a substitute, mask of depression and 'antidote' of narcissism. Eur Proc Soc Behav Sci 31: 986-994, 2017.

47. Richeldi L, Collard HR and Jones MG: Idiopathic pulmonary fibrosis. Lancet 389: 1941-1952, 2017.

48. Raghu G, Amatto VC, Behr J and Stowasser S: Comorbidities in idiopathic pulmonary fibrosis patients: A systematic literature review. Eur Respir J 46: 1113-1130, 2015.

49. Mucsi I, Molnar MZ, Ambrus C, Szeifert L, Kovacs AZ, Zoller R, Barótfi S, Remport A and Novak M: Restless legs syndrome, insomnia and quality of life in patients on maintenance dialysis. Nephrol Dial Transplant 20: 571-577, 2005.

50. Tuncel D, Orhan FO, Sayarlioglu H, Isık IO, Utku U and Dinc A: Restless legs syndrome in hemodialysis patients: Association with depression and quality of life. Sleep Breath 15: 311-315, 2011.

51. Takaki J, Nishi T, Nangaku M, Shimoyama H, Inada T, Matsuyama N, Kumano H and Kuboki T: Clinical and psychological aspects of restless legs syndrome in uremic patients on hemodialysis. Am J Kidney Dis 41: 833-839, 2003.

52. Ohayon MM, O'Hara R and Vitiello MV: Epidemiology of restless legs syndrome: A synthesis of the literature. Sleep Med Rev 16: 283-295, 2012.

53. Stefanidis I, Vainas A, Dardiotis E, Giannaki CD, Gourli P, Papadopoulou D, Vakianis P, Patsidis E, Eleftheriadis T, Liakopoulos V, et al: Restless legs syndrome in hemodialysis patients: An epidemiologic survey in Greece. Sleep Med 14: $1381-1386,2013$

54. Turk AC, Ozkurt S, Turgal E and Sahin F: The association between the prevalence of restless leg syndrome, fatigue, and sleep quality in patients undergoing hemodialysis. Saudi Med J 39: 792-798, 2018

55. Zadeh Saraji N, Hami M, Boostani R and Mojahedi MJ: Restless leg syndrome in chronic hemodialysis patients in Mashhad hemodialysis centers. J Renal Inj Prev 6: 137-141, 2016.

56. Azar SA, Hatefi R and Talebi M: Evaluation of effect of renal transplantation in treatment of restless legs syndrome. Transplant Proc 39: 1132-1133, 2007.

57. Pizza F, Persici E, La Manna G, Campieri C, Plazzi G, Carretta E, Cappuccilli ML, Ferri B, Stefoni S and Montagna P: Family recurrence and oligo-anuria predict uremic restless legs syndrome. Acta Neurol Scand 125: 403-409, 2012.

58. Micozkadioglu H, Ozdemir FN, Kut A, Sezer S, Saatci U and Haberal M: Gabapentin versus levodopa for the treatment of restless legs syndrome in hemodialysis patients: An open-label study. Ren Fail 26: 393-397, 2004

59. Razazian N, Azimi H, Heidarnejadian J, Afshari D and Ghadami MR: Gabapentin versus levodopa-c for the treatment of restless legs syndrome in hemodialysis patients: A randomized clinical trial. Saudi J Kidney Dis Transpl 26: 271-278, 2015.
60. Sagheb MM, Dormanesh B, Fallahzadeh MK, Akbari H, Sohrabi Nazari S, Heydari ST and Behzadi S: Efficacy of vitamins $\mathrm{C}, \mathrm{E}$, and their combination for treatment of restless legs syndrome in hemodialysis patients: A randomized, double-blind, placebo-controlled trial. Sleep Med 13: 542-545, 2012.

61. Cikrikcioglu MA, Hursitoglu M, Erkal H, Kinas BE, Sztajzel J, Cakirca M, Arslan AG, Erek A, Halac G and Tukek T: Oxidative stress and autonomic nervous system functions in restless legs syndrome. Eur J Clin Invest 41: 734-742, 2011.

62. Giannaki CD, Hadjigeorgiou GM, Karatzaferi C, Maridaki MD, Koutedakis Y, Founta P, Tsianas N, Stefanidis I and Sakkas GK: A single-blind randomized controlled trial to evaluate the effect of 6 months of progressive aerobic exercise training in patients with uraemic restless legs syndrome. Nephrol Dial Transplant 28: 2834-2840, 2013.

63. Saha L: Irritable bowel syndrome: Pathogenesis, diagnosis, treatment, and evidence-based medicine. World J Gastroenterol 20: 6759-6773, 2014

64. Wajszilber D, Santiseban JA and Gruber R: Sleep disorders in patients with ADHD: Impact and management challenges. Nat Sci Sleep 10: 453-480, 2018.

65. Thomas R, Sanders S, Doust J, Beller E and Glasziou P: Prevalence of attention-deficit/hyperactivity disorder: A systematic review and meta-analysis. Pediatrics 135 e994-e1001, 2015.

66. Chervin RD, Archbold KH, Dillon JE, Pituch KJ, Panahi P, Dahl RE and Guilleminault C: Associations between symptoms of inattention, hyperactivity, restless legs, and periodic leg movements. Sleep 25: 213-218, 2002

67. Chaiard J and Weaver TE: Update on research and practices in major sleep disorders: Part II-Insomnia, Willis-Ekbom disease (Restless Leg Syndrome), and narcolepsy. J Nurs Scholarsh 51: 624-633, 2019.

68. Didato G, Di Giacomo R, Rosa GJ, Dominese A, de Curtis M and Lanteri P: Restless legs syndrome across the lifespan: Symptoms, pathophysiology, management and daily life impact of the different patterns of disease presentation. Int J Environ Res Public Health 17: 3658, 2020.

69. Anguelova GV, Vlak MHM, Kurvers AGY and Rijsman RM: Pharmacologic and nonpharmacologic treatment of restless legs Syndrome. Sleep Med Clin 13: 219-230, 2018.

70. Garcia-Borreguero D, Silber MH, Winkelman JW, Högl B, Bainbridge J, Buchfuhrer M, Hadjigeorgiou G, Inoue Y, Manconi M, Oertel W, et al: Guidelines for the first-line treatment of restless legs syndrome/Willis-Ekbom disease, prevention and treatment of dopaminergic augmentation: A combined task force of the IRLSSG, EURLSSG, and the RLS-foundation. Sleep Med 21: 1-11, 2016.

71. Trenkwalder C, Winkelmann J, Oertel W, Virgin G, Roubert B and Mezzacasa A; FCM-RLS Study Investigators: Ferric carboxymaltose in patients with restless legs syndrome and nonanemic iron deficiency: A randomized trial. Mov Disord 32: 1478-1482, 2017.

72. Trenkwalder C, Allen R, Högl B, Clemens S, Patton S, Schormair B and Winkelmann J: Comorbidities, treatment, and pathophysiology in restless legs syndrome. Lancet Neurol 17: 994-1005, 2018.

73. Avni T, Reich S, Lev N and Gafter-Gvili A: Iron supplementation for restless legs syndrome-A systematic review and meta-analysis. Eur J Intern Med 63: 34-41, 2019.

74. Akpinar S: Treatment of restless legs syndrome with levodopa plus benserazide. Arch Neurol 39: 739, 1982.

75. Benes H, Kurella B, Kummer J, Kazenwadel J, Selzer R and Kohnen R: Rapid onset of action of levodopa in restless legs syndrome: A double-blind, randomized, multicenter, crossover trial. Sleep 22: 1073-1081, 1999.

76. Trenkwalder C, Garcia-Borreguero D, Montagna P, Lainey E, de Weerd AW, Tidswell P, Saletu-Zyhlarz G, Telstad W and Ferini-Strambi L; Therapy with Ropiunirole; Efficacy and Tolerability in RLS 1 Study Group: Efficacy and Tolerability in RLS 1 Study Group. Ropinirole in the treatment of restless legs syndrome: Results from the TREAT RLS 1 study, a 12 week, randomised, placebo controlled study in 10 European countries. J Neurol Neurosurg Psychiatry 75: 92-97, 2004.

77. Collado-Seidel V, Kazenwadel J, Wetter TC, Kohnen R, Winkelmann J, Selzer R, Oertel WH and Trenkwalder C: A controlled study of additional sr-L-dopa in L-dopa-responsive restless legs syndrome with late-night symptoms. Neurology 52: 285-290, 1999 . 
78. Allen RP and Earley CJ: Augmentation of the restless legs syndrome with carbidopa/levodopa. Sleep 19: 205-213, 1996.

79. Wijemanne S and Ondo W: Restless Legs Syndrome: Clinical features, diagnosis and a practical approach to management. Pract Neurol 17: 444-452, 2017.

80. Picchietti DL, Hensley JG, Bainbridge JL, Lee KA, Manconi M, McGregor JA, Silver RM, Trenkwalder C and Walters AS; International Restless Legs Syndrome Study Group (IRLSSG): Consensus clinical practice guidelines for the diagnosis and treatment of restless legs syndrome/Willis-Ekbom disease during pregnancy and lactation. Sleep Med Rev 22: 64-77, 2015.

81. Beneš H, García-Borreguero D, Ferini-Strambi L, SchollmayerE, Fichtner A and Kohnen R: Augmentation in the treatment of restless legs syndrome with transdermal rotigotine. Sleep Med 13: $589-597,2012$.

82. Garcia-Borreguero D, Cano-Pumarega I and Marulanda R: Management of treatment failure in restless legs syndrome (Willis-Ekbom disease). Sleep Med Rev 41: 50-60, 2018.

83. Comella CL: Treatment of restless legs syndrome. Neurotherapeutics 11: 177-187, 2014.

84. Prosperetti C and Manconi M: Restless legs syndrome/willis-ekbom disease and pregnancy. Sleep Med Clin 10: 323-329. xiv, 2015.

85. Telstad W, Sørensen O, Larsen S, Lillevold PE, Stensrud P and Nyberg-Hansen R: Treatment of the restless legs syndrome with carbamazepine: A double blind study. Br Med J (Clin Res Ed) 288: 444-446, 1984.

86. Garcia-Borreguero D, Larrosa O, de la Llave Y, Verger K, Masramon X and Hernandez G: Treatment of restless legs syndrome with gabapentin: A double-blind, cross-over study. Neurology 59: 1573-1579, 2002.

87. Jiménez-Jiménez FJ, Alonso-Navarro H, García-Martín E and Agúndez JAG: Treatment options for idiopathic restless legs Syndrome. Eur Neurol Rev 10: 45-55, 2015.
88. Walters AS, Wagner ML, Hening WA, Grasing K, Mills R, Chokroverty $\mathrm{S}$ and Kavey N: Successful treatment of the idiopathic restless legs syndrome in a randomized double-blind trial of oxycodone versus placebo. Sleep 16: 327-332, 1993

89. Walters AS, Winkelmann J, Trenkwalder C, Fry JM, Kataria V, Wagner M, Sharma R, Hening W and Li L: Long-term follow-up on restless legs syndrome patients treated with opioids. Mov Disord 16: 1105-1109, 2001.

90. Montagna P, Sassoli de Bianchi L, Zucconi M, Cirignotta F and Lugaresi E: Clonazepam and vibration in restless legs syndrome. Acta Neurol Scand 69: 428-430, 1984.

91. Saletu M, Anderer P, Saletu-Zyhlarz G, Prause W, Semler B, Zoghlami A, Gruber G, Hauer C and Saletu B: Restless legs syndrome (RLS) and periodic limb movement disorder (PLMD): Acute placebo-controlled sleep laboratory studies with clonazepam. Eur Neuropsychopharmacol 11: 153-161, 2001.

92. Xu XM, Liu Y, Jia SY, Dong MX, Cao D and Wei YD: Complementary and alternative therapies for restless legs syndrome: An evidence-based systematic review. Sleep Med Rev 38:158-167, 2018.

93. Einollahi B and Izadianmehr N: Restless leg syndrome: A neglected diagnosis. Nephrourol Mon 6: e22009, 2014.

94. Harrison EG, Keating JL and Morgan PE: Non-pharmacological interventions for restless legs syndrome: A systematic review of randomised controlled trials. Disabil Rehabil 41: 2006-2014, 2019.

This work is licensed under a Creative Commons Attribution-NonCommercial-NoDerivatives 4.0 International (CC BY-NC-ND 4.0) License. 